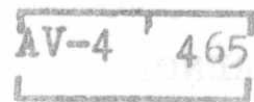

$\Gamma$

\title{
Two Adaptive Algorithms for Target Detection in Cluttered lwases
}

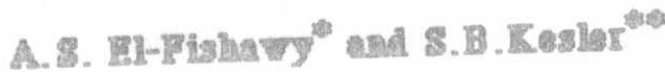

\section{ABSTRACT}

Datection of ungets in in a bow signal to clutter plus noise ratio (SCNR) is a problem of increasing interest. In this pager wo present two edaptiwe algorithms for the dotection of small tragets (of the oxder of one pixel) in images using reference comelated fromes (the reference frames can be obtained either from frequency bands of the same scene or from different sequential observation in time) in a bw signal to chuter plus noise ratio (\$CNR) environment (of the onder of $-14.5 \mathrm{AB}$ ). They both have the ability to track the nonstationary image signals (turges and clutter plus noise) and suppress the chuter plus noise background. Both detectons ane bsed on time varying aucregressive models bo model mage bacikground and on correlation canceling concept. The first one uses an order recurstpe least squares (ORLS) lattice fliper, while the sacond one is based on a normalized version of tha two dimensional least moan square (TDLMS) algorthon. The haflueses of the oxlex of the detectors on their detaction perfomance is studied. The perfomance of the two elgotiturns are evaluated using an optisal satelune image, as a chuter beckground, wth computar generated target and noise added o it.

\footnotetext{
* Assistant Professor, Dpt. of Communications Eng., Menoufia Unifersity, Menouf, Menoufia, Egypt. **. Associate Professor, Dpt. of Electrical and Computer Eng. , Drexel University, Philla, Pa 19104, USA
} 


\section{INTRODUCTION}

Detection of unfriendly targets is an integral part of the air, ground and sea defense. The area under surveillance is monitored by a set of receivers, active andlor passive. The receivers (sensors) are intercepting the energy which is reflected from, andfor radiaved by targets of interest. The received signal, in addition to the desired information, usually contains heavy clutter and background noise. In such environment, target detection procedunes should be able to isolate the moving target in signal-to-clutter-plus-noise ratios (SCNR) in the order of -10 to $-20 \mathrm{~dB}$. Clutter sounces themselves are not entirely spatially stationary, even during the relatively short time spans. Weather clutter is slowly moving due to wind activities, and clear air turbulence, among other causes. Even ground cluter is parying with the local movement of regetation. Sea clutter exhibits various statistical characteristics in different sea states.

Gogliari et. Al [1] have developed an algorithm for the devection of optical targets by using comelated reference scene. However, this algorithm demands a substantial computation time, most of which is used for estimating the corrance matrix and for calculating matrix inverses. Margalit ot. al [2] have developed a detection algorithm which is based on the assurziption that the image intensity is spatially Gaussian process and that the covariance matrix of optical and infrased (IR) images, after subtraction of correct variable mean, is approximately a constant times an identity matix. Therefore, the resulting Gaussian image processes are nearly White. This algorithm wros shown to save most of the computation time requined by the optimum algorithm developed in [1]. In addition, Chen and Reed [3] have developed an alvemate algonithm for the detection of optical target by using a reference scente. The above algorithms are based on the approximation of the image data by a white Gaussian process. For real images, this assumption is not necessarily valid in general case.

To remedy the mentioned problerns, we suggest two adaptive algovithms for cluter plus noise suppression and opticallinfroned targets detection. The first one is based on the order recursive least-squares (ORLI) lattice algorithm $[4,5]$, while the second processor uses a nomalized version of the two dimensional least mean square algorithm (TDLMS) $[5,6]$

\section{PROBLEM FORMULATION}

Let us assuma that we have two $M$ by $N$ images of a specific scene that are viken at different times. These images can be forwand looking infrared (PLIR), radar echo, remoie sensing. The first (primary) image, d(m, $n$ ), differs from the second (zeference) one, $x\left(m^{\prime}, n\right)$, in that it has a terrget embedded in a background clutter. The $\mathrm{m}(=0,1, \ldots, \mathrm{M}-1)$ and $\mathrm{n}(=0,1, \ldots, \mathrm{N}-1)$ ane the spatial coontinates of the pixel location with respect o some prespecified coordinats system, say, with the origin in the upper left comer. The background in the nefeneric image may be shifted or blurred and may be of different brightoess compared to the background in the primary ons. The backgrounds, however, numain spatially correlated, while independent of arget component.

Our objective is to design alapdive burget defoctor that shovld have the ability to treck the nanstationary image signals, suppress the background and efficiently de tect the changes (targes) between two images in a sequence in a bV signal to cluter plus noise ratio (of the order of $-14.5 \mathrm{~dB}$ ). Tanget image edges should be praserved so that relatively small size target can be discerned. In the applications where images appear in sequence, both adaptive algorithms can be applied to a poir consisting of s cunzent frame and a reforence one.

General block disgram of the suggesed target detectors is shown in Fig.1. Signals and processor parametrs ane given as 1-D functions of spatial coord inates as they will be used in the context of the ORL.S processor. For the origin in the upper left comer of $\mathrm{M}$ by $\mathrm{N}$ image, the value of the singlo spatial coondinate $\mathrm{n}$ is rolated in $\mathrm{m}$ and n \&:

$$
n=m^{\prime} N+n^{\prime} ; n^{\prime}=0, \ldots, N-1, m^{\prime}=0, \ldots, \mathbb{M}-1
$$

and $n$ runs from 0 to MN-1. Thus, for the "zerotu" $m$. 
$\mathrm{n}^{\prime}(-0,1, \ldots, \mathrm{N}-1)$ are the spatial coonitinates of the pixel location with respect to some prespecified coondinate sysiem, say, with the origin in the upper left comer. The background in the reference image may be shifted or blurred and may be of different brightness companed to the background in the primary one. The backgroumds, however, nemain spatially comelated, while independent of target component.

Our objective is to design adaptwe target detector that should have the ability to track the nonstationary image signals, suppress the background and efficiently detect the changes (targets) between two images in a sequence in a low signal to clutter plus noise ratio (of the onder of $-14.5 \mathrm{~dB}$ ). Target image edges should be preservad so that relatively small size trget can be discemed. In the applications where images appear in sequence, both adaptive algorithns can be applied to a pair consisting of a current frame and a reference one.

General block diagram of the suggested trrget detectors is shown in Fig. 1. Signols and processor parameters ane given as 1-D functions of spatial coordinates as they will be used in the context of the ORLS processor. For the ongin in the upper left comer of $\mathrm{M}$ by $\mathrm{N}$ imege, the valve of the single spatial coordinate $\mathrm{n}$ is related to $\mathrm{m}$ and
$\mathrm{n}$ ' $\mathrm{s}$ :

$$
n^{\prime}=m^{\prime} N+n^{\prime} ; n^{\prime}=0, \ldots, N-1, m^{\prime}=0, \ldots, M-1
$$

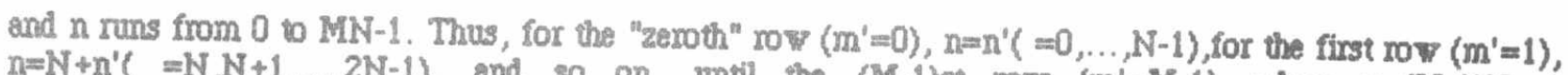
$n=N+n^{\prime}(=N, N+1, \ldots, 2 N-1)$, and so on, until the $(M-1) s t$ now $\left(m^{\prime}=M-1\right)$ where $n=(M-1) N+n^{\prime}$ $(=\mathrm{MN}-\mathrm{N}, \mathrm{MN}-\mathrm{N}+1, \ldots, \mathrm{MN}-1)$. The 1-D representation for the ORLS processor is adequate since it converges rapidly for even the moderate level of 1-D crossconrelation between horizontal lines in two images. For the TDLMS aigorithm, the block diagram would be the same, except that all the signals and filter parameters would be two-dimensional functions of spatial coordinates instead of one-dimensional. The primary image input d(n) (necent frame) consists of the desired arget signal $(n)$ embedded in a clutter background $s(n)$ and a colored noise $v_{1}(n)$. In toms of Wiener fillering theory, $d(n)$ represents the desired nesponse. The reference input $x(n)$ consists of aclutter (possibly blunred), sy $(n)$, and a coloned noise, $q_{2}(n)$, which is spatially comelated with that of the primary image. The colored noises $\psi_{1}(n)$ and $v_{2}(n)$ can be considered as additional clutter sounces.

Defining the $L-$ th order fllter coefficient wector by $=\left[w_{0}, W_{1}, \ldots, W_{L-1}\right]^{T}$ and the $L x 1$ reference input vector as $z(n)=[x(n), x(n-1), \ldots, x(n-L+1)]^{T}$, the output of the target detecior in Pig. 1, at spatial coondinate $n$, is given by

$$
Y(n)=d(n)-z(n)=d(n)-v^{T} T_{X}(n)
$$

where the primary input is

$$
d(n)=(n)+z(n) \text { and } z(n)=s(n)+\psi_{1}(n)
$$

is the clutter plus noise component of primary input and (n) is the target signal component embedded in the

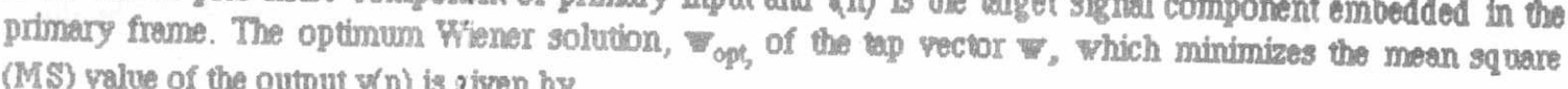
(MS) value of the output $y(n)$ is given by

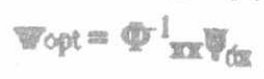

where $\Phi_{\mathrm{mz}}$ is the reference input atocovariance matdx given by

$$
\text { e }=\mathrm{E}\left[\mathrm{X}(\mathrm{n}) \mathrm{z}(\mathrm{n})^{\mathrm{T}}\right]
$$

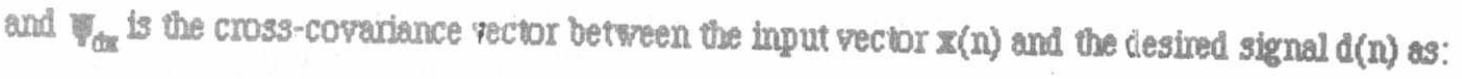

$$
\nabla_{\text {tax }}=\mathrm{E}[\mathrm{d}(\mathrm{n}) \mathrm{z}(\mathrm{n})] \text {. }
$$
The "minimum" output of the change defector, that is, the output having mininized energy, which comesponds
to the optimum filter is 


\section{AV-4 468}

autocovariance matrix, $\Phi(n)$, of the reference input vector, $\mathbf{z}(n)=[x(n), x(n-1), \ldots, x(n-1+1)]^{T}$, and the $L x 1$ cross-covariance vector, $\Psi(n)$, between $x(n)$ and the desired input, $d(n)$, as follows $[8]$ :

$$
\begin{aligned}
& \Phi(n)=\Phi(n-1)+\sum_{i=0}^{L-1} \sum_{j=0}^{L-1} x(n-1) \mathbf{x}(n-j) \\
& \Psi(n)=\psi(n-1)+\sum_{j=0}^{L-1} d(n) \mathbf{x}(n-j)
\end{aligned}
$$

After $\Phi(n)$ and $\Psi(n)$ are updated for a given $n$, the reflection coefficients $K_{m} f_{m}(n), K_{m}(n)$ and $H_{m}(n)$ are onder-updated using $E_{m-1,0,0}(n), R_{m-1,0,0}(n), C_{m-1,0,}(n)$ and $D_{m-1,0,}(n)$, and the GRE's of order $m$ are obtained by using the values at $m-1$, and the reflection coefficients of order $m$. Once the adaptation is perfomed, the signal $y_{\min }(n)$ represents the difference image, i.e., $y_{\min }(n)=g_{1}(n)=\psi(n)$, which contains the target to be detected.

\section{The TDLMS Target Detector:}

The 2-D counterpart of the WHidrow LMS algorithm was originally intoduced by Hadhoud and Thomas [6] for handling 2-D signals. It is well suited for our problem because of the assumption of image background correlatedness in both dimensions. The TDLMS algorithm employs the method of the steepest decent, and the cument filter weight matrix is given by the previous weight matrix plus a change proportional to the negative gradient of the emor power. Refeming to Fig.4, the 2-D amays $\mathrm{z}$ and $\mathrm{d}$ are the reference and primary inputs, respectively, both of dimension $M$ by $N$. The filter weight matrix is of dimension $\mathrm{K}$ by $\mathrm{L}$. In the example in Fig. $4, M=5, N=10, K=2$ and $L=5$. Of course, the $2-D$ algorithm reduces to its $1-D$ counterpart for $K=1$. When the filter is operating on the neighborhood of the pixel $x(m, n)$, as indicated in Fig.4, the error signai at the $j$-th iteration, $y(m, n)$ is given by

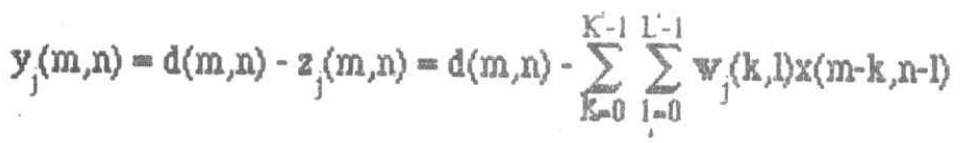

In the above equation mruns from 0 in $M-1$ and $n$ runs from 0 to N-1. Limits $K^{\prime}$ and $L^{\prime}$ are equal, respectively, to $K$ and $L$ for $m \geq K-1$ and $n \geq L-1$. Towards the image boundaries, where $m<K-1$ andlor $n<L-1, K$ and $L$, reduce, respectively to $\mathrm{m}$ and $\mathrm{n}$. When the filter window scans the image left- 0 -right, and op-n-botom iteration index is related to the current pixel coondinates by

$$
j=m N+n \quad n=0, \ldots, N-1 \text { and } m=0, \ldots, M-1
$$

The weight adjustment algorithm is then given by

$$
\nabla_{j+1}(k, l)=\nabla_{j}(k, l)+2 \mu y_{j}\left(m^{\prime}, n^{\prime}\right) \times\left(m^{\prime}-k, n^{\prime}-1\right),
$$

The value of $\mu$ is chosen to balance the tradeoff between the convergence speed, tracking ability and steady-state MSE. To optimize the convergence factor $\mu$, different ways were sugessted for 1-D gradient-based adaptive algorithms. We use a normalized value of $\mu[9,10]$, 1.e., 
1. The sugessted trget detector could suppress the chuter and noiso background components vhile heephig the tanget power unatenuated.

2. The Tiener solution nequines prior information about the covariance matrix of the chutter plus noise. However, in most practical situations, that required statistics is seldom knovn in advance, and typically, it veries with time. Therefore, the most effective solution for met detection relies on the use of adaptive filters.

\section{ADAPTIV ALCORITHIS FOR TARGET DRTECTON}

Assume arallable a recent frame, $d(n)$, which contains a arget embedded in a clutier plus noise background. Adaptive anget desectors can be developed by using information that the clutter signals in the necent frame and in a raterence trame are spatilly conolated.

The error signal $Y(n), Y(m, n)$ in the case of TDLMS algorithm, is used to generate the performance criterion for adjusting the filter coefficients. The adaptive filter (ORL 8 latice or TDLMS) uses the reference input frame to estimate the clutter plus noise background in the primary Input frame. Then, the estimated cluter plus noise is subtracted from the primary input, leaving only the estimate of the target which we wish to detect.

The perfornance criterion for the TDLMS is to minimize the least mean square of value $Y^{\prime}(m$ ', $a$ '), as the name implies. On the other hand, the exact least squares (ELS) algorithms perform the minimization of the finite sum. of $L$ values of $X(n)^{2}$ which is known exactly for each step $\mathrm{n}$. Here, we theat the wariant of $\mathrm{EL} \mathrm{S}, \mathrm{known}$ as the order-recursive least squares (ORLS) lattice elgorithm.

\section{The Adaptive ORLS Algorithm}

Fig.2 shows the foint lattice filwer of ouder $\mathrm{L}$, Fhere $\mathrm{e}_{\mathrm{n}}(\mathrm{n})$ and $\mathrm{r}_{\mathrm{M}}(\mathrm{n})$ ane, nespectively, the forwand and backswand nesidual at spatial bcation $\mathrm{n}$, of the inverse filter part of the latice, corresponding time-varing werlection coefricients are $\mathrm{K}_{\mathrm{m}}(n)$ and $\mathrm{K}^{\mathrm{s}}$ (n). The joint path input $\mathrm{g}_{0}(n)$ is the primary image $\mathrm{d}(n)$, whereas the reference inage $\mathrm{z}(\mathrm{n})$ is fed to the inverse filer input. The backwand nesidvals $\mathrm{I}_{\mathrm{a}}(\mathrm{n})$ ane veighted by joint redection coelficients $H_{m}(n)$ to obtain the estimates, $z(n)$, of the chuther plus noise component, $z(n)$, of the

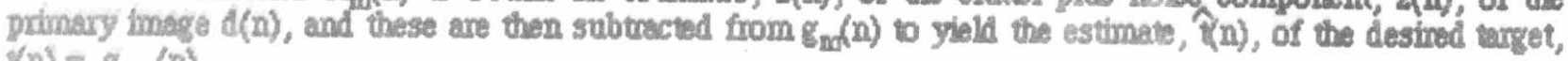
$(n)=g_{L-}(n)$.

We vise the ORLS lattice algorithm for computing the reflection and joint coefficlents of the lattice rilter. This algorthm differs from the conventional LS lattice [7] in that the time and order recursions ane separated as shown in Fig. 3. The problem of poor initial speed of convergence of the mired time and order recursive Ls lattice algorithms and the related gradient lattice algorithms in a fired-point environment have been alleviated. Since this approech does not requine residual necursions, encor accumulation does not occur, and hence there is no degrededon of the sdaptation speed.

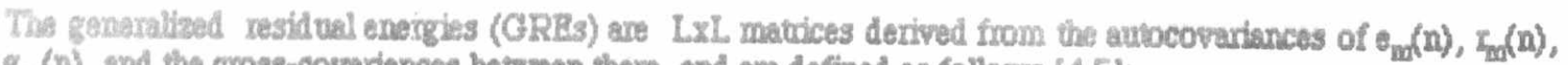
$\mathrm{F}_{\mathrm{m}}(\mathrm{n})$, and the cross-covariances between them, and are defined as follows $[4,5]$ :

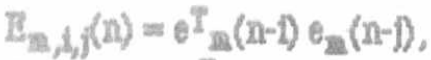

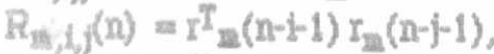

$$
\begin{aligned}
& C_{m, j}(n)=0^{T} a_{n}(n-j-1) r_{n}(n-j-1) \text {, }
\end{aligned}
$$

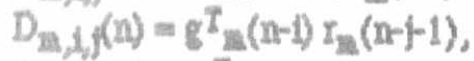

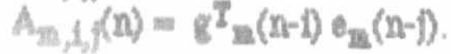

where $0 \leq \mathrm{i}$ if $5 \mathrm{~L}-1$. At each pixel bcation $\mathrm{n}$, "the covariance matrix tracking" block of Fig. 3 updates the LxL. 


$$
\mu_{j}=\frac{\mu_{0}}{(L K)\left(\sigma_{j}^{2}\right)}, \quad 0<\mu_{0}<1
$$

whene LK is the number of filter coefficients and $\sigma^{2} \mathrm{j}$ is the power estimate of the reference frame which is updated for the cument input pixel value $x(m, n)$ as follows

$$
\sigma_{j}^{2}=\alpha x^{2}(m, n)+(1-\alpha) \sigma_{j-1}^{2}, \quad 0 \leq \alpha<1
$$

and $\alpha$ is a forgetting factor. This estimate fits nonstationary environment assumption since $\alpha$ can be selected to reduce the influence of past input pixels. The initial value of $\sigma^{2}$ is the sf furiuj estimate of the input power of the refenence frame $x(m, n)$. In many application, images are of window size is valsen to be square, resulting in $\mathrm{K}=\mathrm{L}$.

\section{COMPUTER SIMULATION RESULTS}

In an experimental verification of the two adaptive target detectors, an optical satellite image $s(n)$ of a city area, of size $42 \times 42$ as shown in Fig.5, is used as a clutter background for both the primary input, $\mathrm{d}(\mathrm{n})$, and the reference input, $x(n)$. The primary input is formed from $s(n)$ by adding on it a colored noise, $\varphi_{1}(n)=a_{1} \psi_{1}(n-1)+$ $\bigvee(n)$ where $\mathfrak{V}(n)$ is zero mean white Gaussian noise of wariance $\sigma$ and a one pixel target centered at spatial location $m=14, n=24$. Therefore,

$$
d(n)=v(n)+2(n) \text {, where } 2(n)=g(n)+v_{1}(n)
$$

Such a composite primary imsge input is shown in Fig. 7. The reference image input, $x(n)$, is a blumed version of $s(n)$ with a colored noise $\psi_{2}(n)$ (correlated with $\nabla_{1}(n)$ ) as shown in Fig.8. Bluming is perfomed by passing $s(n)$ through a low-pass filier, e.g,

$$
s_{b}(n)=0.95 s_{b}(n-1)+0.05 s(n)
$$

Therefore,

$$
x(n)=s_{b}(n)+\psi_{2}(n)
$$

where $v_{2}(n)=q_{Q} v_{2}(n-1)+v_{(2}(n)$ and $v(n)$ is white gaussian noise. Is can be shown that the crosscorellation between $v_{1}(n)$ and $\psi_{2}(n), \psi_{v \mid}(k)$, is

$$
W_{\nabla_{1}, 2}(k)=\frac{a_{1}^{k}}{1-a_{1} a_{2}} \sigma_{n}^{2}, \quad k \geq 0
$$

and the correlation coefficient, $I_{\mathrm{q} \mid \mathrm{v}}(\mathrm{k})$, will be

$$
r_{\nabla_{1} \nabla_{2}}(k)=\frac{\Psi_{\nabla_{1} \nabla_{2}}(k)}{\sqrt{\sigma_{\nabla_{1}}^{2} \sigma_{\nabla_{2}}^{2}}} \sigma_{\mathrm{n}}^{2}
$$


Sustituting with equation (6) in equation (7), the comelation cocficient for zero lag, $\mathfrak{r}_{\text {vl }}(0)$, will be

$$
r_{v_{1} v_{2}}(0)=\frac{\sqrt{\left(1-a_{1}^{2}\right)\left(1-a_{2}^{2}\right)}}{\left(1-a_{1} a_{2}\right)} \leq 1
$$

We choose $a_{1}=-0.5$ and $a_{2}=0.8$ such that $\Psi_{\text {vif }}(0)$ and $r_{\text {vidz }}(0)$ will be equal 00.7142 and 0.3712 , respectively. To quantify the SCNR in the inpur and outyut images, the power of the clutter plus noise in the primary input $z$ (n) is computed as:

$$
p_{2}=(I M N) \sum_{i=0}^{M N-1}\left(s^{2}(i)+\nabla^{2}(i)\right)
$$

where the pirel location is taken to be in one dimension and the images are of size M by N. Similarly, the power of the target imoge signal is computed:

$$
P_{t}=\left(1 Q^{2}\right) \sum_{i=0}^{Q^{2}-1} t^{2}(i)
$$

where $Q$ is empirically selected size of the square window that encompases the target.

Thus, the input SCNR is given by

$$
\$ C N R_{\mathrm{i}}=\mathrm{P}_{\mathrm{q}} / \mathrm{P}_{\mathrm{z}} \text {, }
$$

The output of the target detector $Y_{\text {min }}(n)$ consists of the estimated arget signal in addicion to the residual clutter plus noise. The output SCNRo is

$$
\operatorname{SCNR}_{0}=\left[\left(1 / Q^{2}\right) \sum_{i=0}^{Q^{2}-1} \operatorname{ch}^{2}(i)\right) /\left((1 / M N) \sum_{i=0}^{M N-1}(X(i)-\operatorname{ch}(i))^{2}\right]
$$

The two adaptive detection algorithms ane applied to the primary and reference images of Pigs.6 and 7,

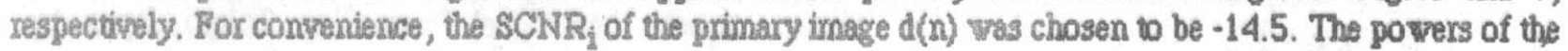
colored noises $v_{1}(n)$ and $\gamma_{2}(n)$ were adjusted so that the clutter to noise ratio (CNR) in the primary input is equal to $24.4 \mathrm{~dB}$. Por the ORLS processing, images are processed row by row sequentially so as to represent the one-dimensional input to a filter of onder $L=2$. The output, $(n)$, of the ORL. detector is shown in Pig. 8. The choice of $\mathrm{L}=2$ for the onder of the latdice was guided by it effect on the improvement factor, which is shown in Fig.9. The optimal value for $\mathrm{L}$ is 4 , however, the difference in performance for any value of $\mathrm{L}$ greater than one all the way up to $\mathrm{L}=10$ is negligibly small. The choice of minimum value in this range speeds up the compuration significantly. The $\mathrm{SCNR}_{0}$ and the improvement factor wrese $28.63 \mathrm{~dB}$ and $43.13 \mathrm{~dB}$, respectively.

The TDLMS detector with a window size of $2 \times 2$ pixels, a convergence factor $\mu_{0}=0.047$, a forgeting factor $\alpha=0.01$, and an initial power estimate of $x(m, n)$ of 0.02 was used to scan the images left-o-night, and top-to-bottom. The resulting output is shown in Fig.10. Again the target is clearly visible, indicating the powerful detection capabilities of the proptsed detectors. Compared to the output of the ORL.S in Pig.4.9, however, the detected target is of a bwer intensity due to a generally slower convergence of the TDLMS. The first rov of the output image shows strong chutter background due to the same effect, combined with the fact that the vindow 'size' is reduced to $2 \times 1$ pixtls. The SCNR and improvement factor values of $8.1 \mathrm{~dB}$ and L 
$22.58 \mathrm{~dB}$, respectively.

\section{CONCLUSIONS:}

The presented results show that botr the suggested adaptive target detectors display good perfomance for the detection of small size varget (of the onder of one pixel) embedded in cluttered images.

Neither vanger desectors requires ary prior infornation about the shape or position or the statistics of the change, or the stetistics of chuter and noise, except that the baclegsound cluter and noise in the primary and reference imeges are spatilly correlatod. Also, the terget imegaltarget and the clutter plus noise beclgrounis ane sasumed to be uncorralised. The beckground in the refenence imege can be scaled and/or blumed andfor have different brightness from the primary one. Both the suggesed change detoctons could successfully track the nonstationary tmage signals, suppress the clutter and noise background and dewect the change betwreen the images in a very bo signal o cluter and noise envinoments (\$CNR of the onder of $-14.5 \mathrm{~dB}$ ).

The order recursivw lase squares (ORL \$) anger detactor; however, is more robust in wery bow signal to clutter ratio environments. It conperges very guickly, within about 2L iterations, and does not require adjustment of any tuning paramaters. It is selativoly insensitive to finite word length effects, and can ossily be implamented by using systolic architecture, so that the computation time can be drastically reduced.

The TDLMS arget detector is computationally as efficiant as its 1-D counterpar, neguires the onder of $2(\mathrm{LxK}$ ) muliplication per teration. Its convergence is, however, slow which may result in insufficient background suppression in the upper-left negion of the image. It needs adjustments of the convergence factor and about 300 to 400 inerations for tise algorthm to converge.

\section{REFTRERCES}

[1] R. M. Gegliandi, I. \$. Resd, H. M. Shoo, and A. Margalit, "Opticel Target Detection Using Dual Scene Observation," Univ. of Southem Califomia, Electrical Engineering, Report, Feb. 1983.

[2] A. Margelit, I. S. Reed, and R. M. Gagliandi, "Adaptive Optical Terget Detection Using Comelated Images," IERE Trans. Aerospece and Electronic Systems, vol. ABD-21, no .3. May 1985, pp 494-405.

(3) J. Y. Chen and I. \$. Reed, "A Detection Algoxithm for Optical Tangets in Clutter," IEEE Trans. Aerospace and Electronic sysams, wol. AEs 53 , no.1, Jan. 1987, pp. 46-59.

[4] P. Strobach, "New Forms of Least Squane Algonithms and Comparison of Their Round Off Enor Characeristics," Proc. of the Int. Con'. Acoust., Speech and signal Processing, Tokyo, Japan, pp. $573-576,1986$.

[5] A. \&. El-Fishawy, "Adaptwe Algorithns for Change Detection in Imsge Sequence," Ph.D Dissertation, Drexel Unwwrsity, Philadephia, PA, U. S.A, May 1990.

[6] M. Hodhoud, and D. Thomas, "The Two Dimensional Adaptive LMS (TDLMS) Algoritum," IEEE Trans. Cincuits and వysams, wol. 35, no. 5, May 1988, pp. 485-494.

[7] E. H. Satorius and J. Pack, "Application of Least Squares Lattice Algonithms to Adaptive Bqualization," IEER Trass. Communication, vol. COM-29, Feb. 1981, pp. 136-142.

[8] A. Giordano and I. Hisu, Least Square Estimation wiơ Application to Digital Signal Processing, Prentice Hall, 1985

[9] W. Milchael and F. Yossa," Optimality in the Choice of the Convengence Factor for Adaptive Algonthrns," Proc. IEEE Int. Symp. on Cincuits and Systems, Newport Beach, CA, 1983, pp. $1376-1370$.

[10] B. Widrow, and S. Stewns, "Adaptive Signal Processing," Prentice-Hall, Englewood Chifs, N.J., 1985. 


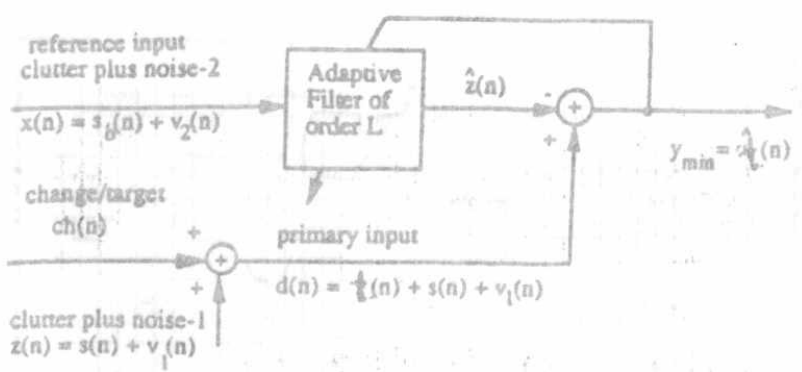

Fig.1 General block diagram of the suggested detectors.

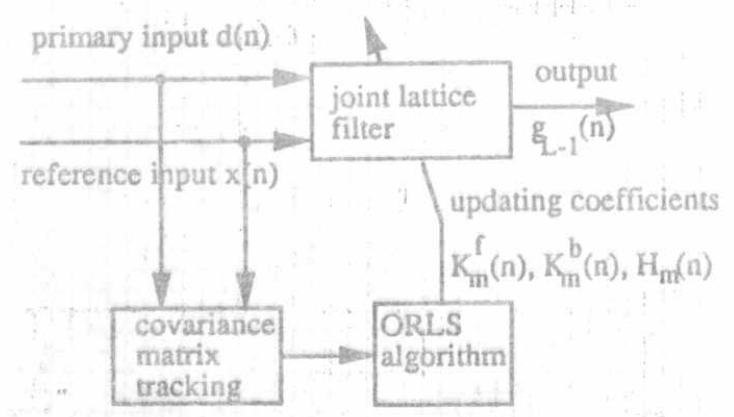

Fig.2 The block diagram of the ORLS lattice aigorithm

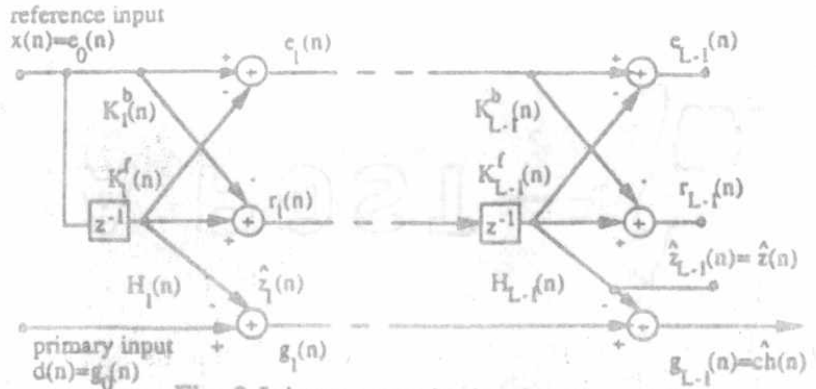

Fig. 3 Joint process lattice for change detection. reference input, $x \quad$ primary input, $d$

$0 . \ldots \quad 0 . \ldots+1$

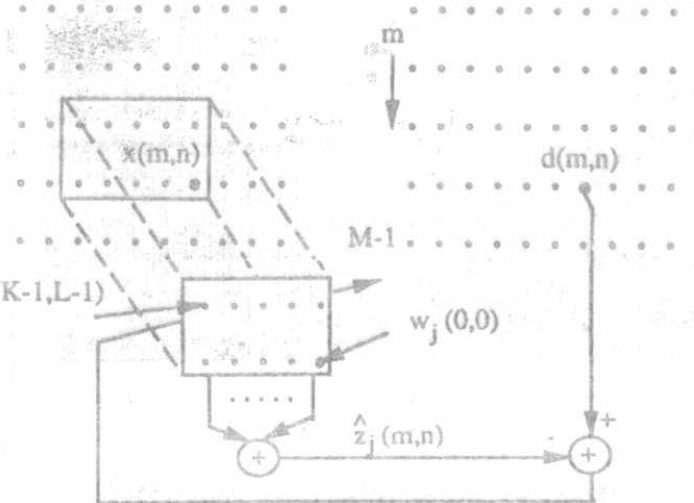

error, y $(\mathrm{m}, \mathrm{n})$

Fig.4 The block diagram of the TDLMS algorithm
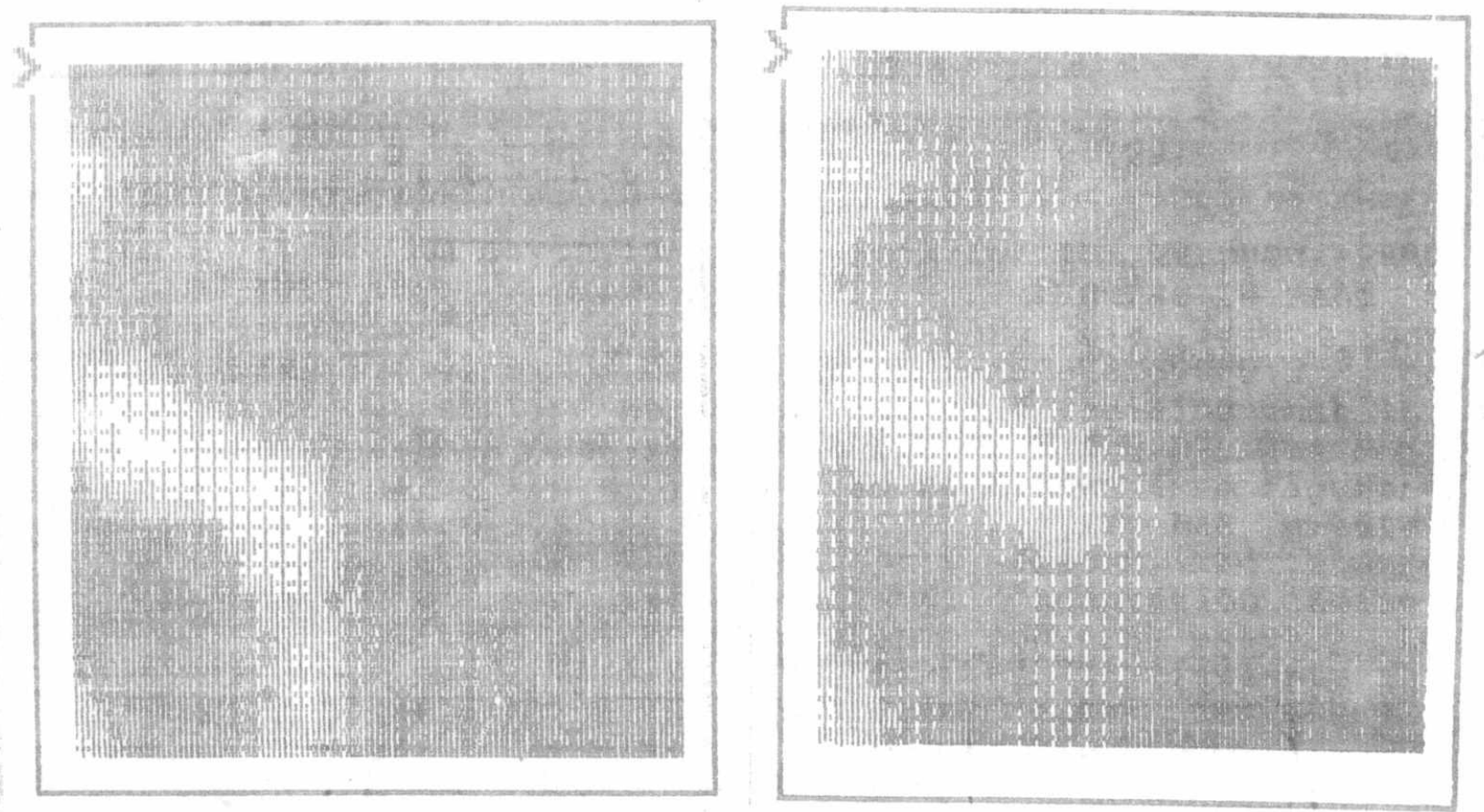

SCNR: $=-14.49 \mathrm{~dB}$

Fig.6 Primary input image, d(n), with a single Fig. 7 Reference input image, $x(\mathrm{n})$. $L$ : pixel target 
$\mathrm{AV}-4 \quad 474$

r
FOURTH ASAT CONFERENCE

14-16 May 1991, CAIRO

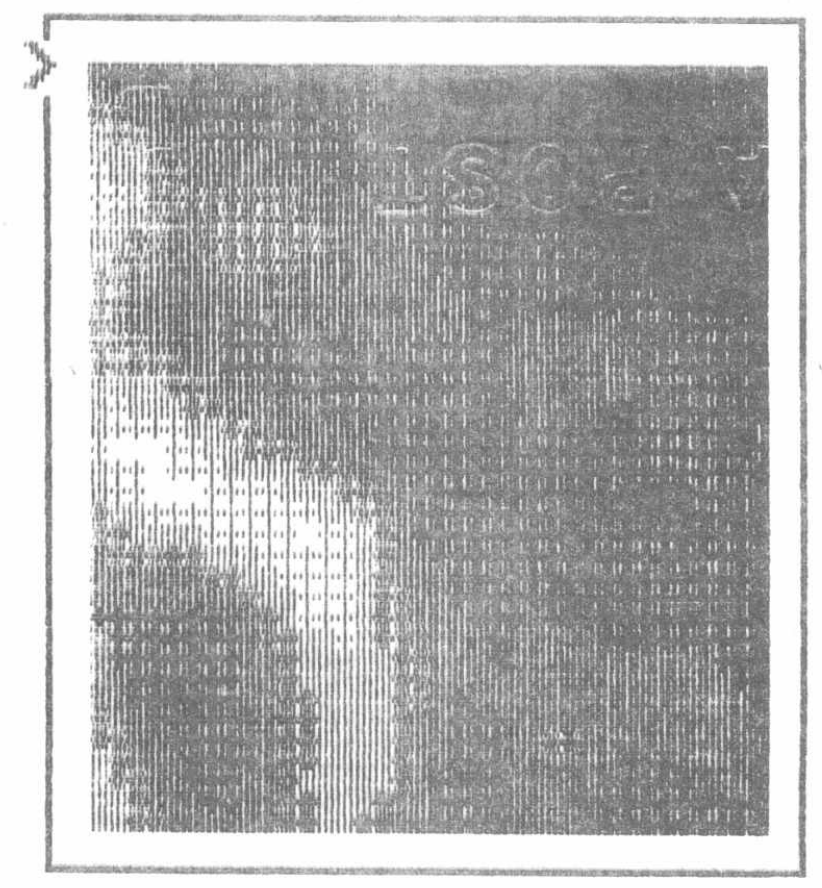

Fig. 5 Optical satellite image, s(n)。

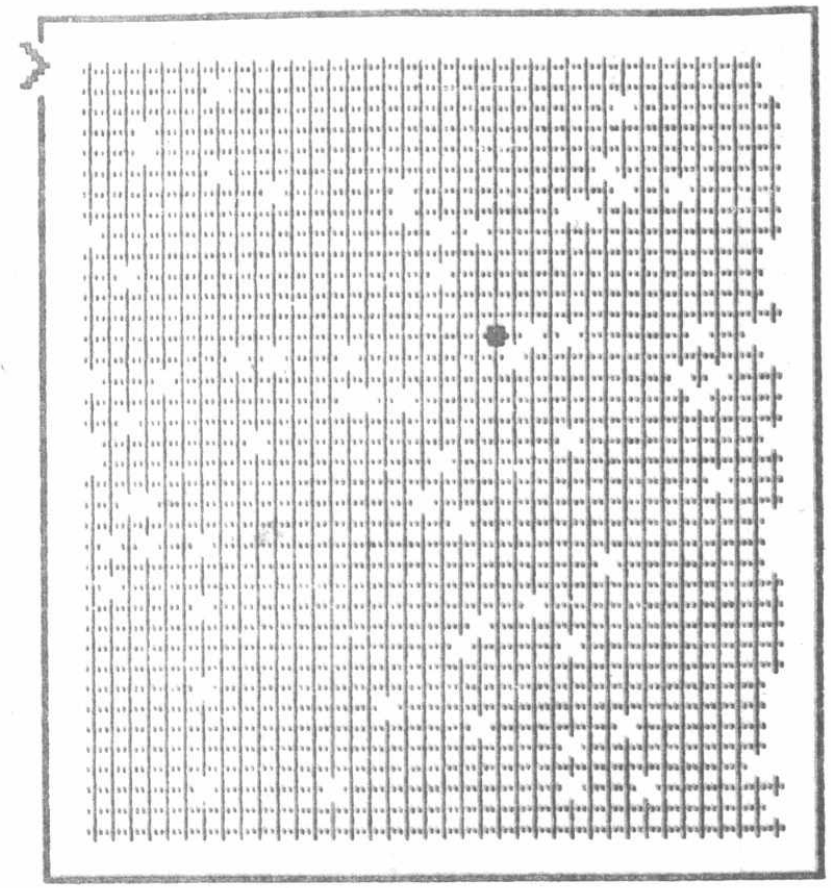

Fig. 8 Output image of the ORLS detector

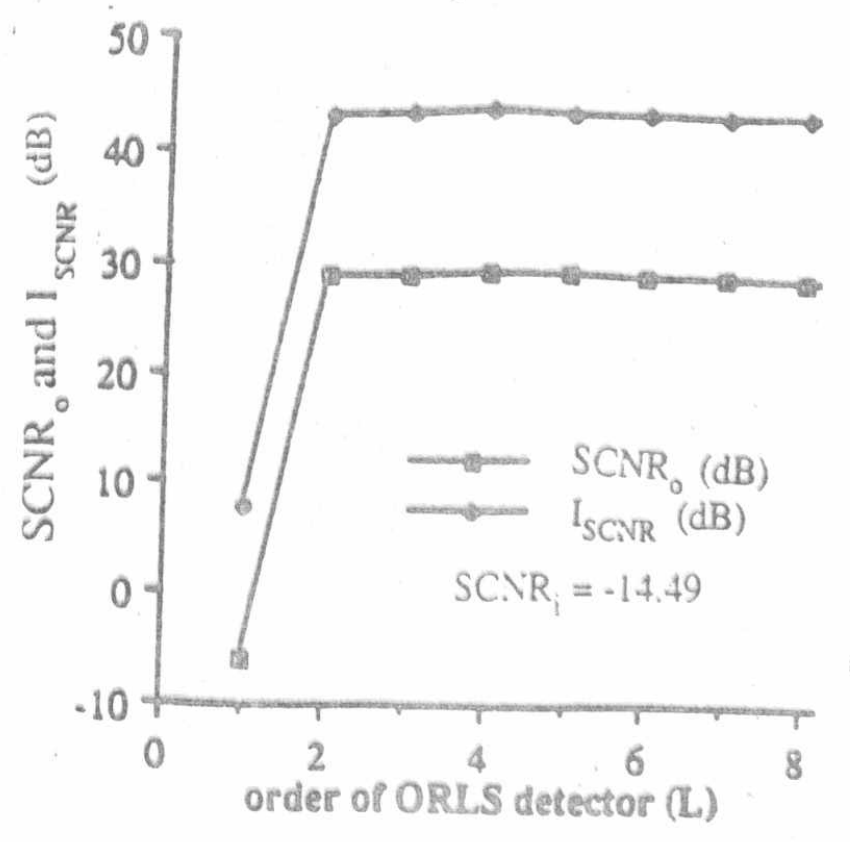

Fig. Effect of the order L of the ORLS lattice on the SCNR and the improvement factor.

Fig. joutput image of the TDLMS detector

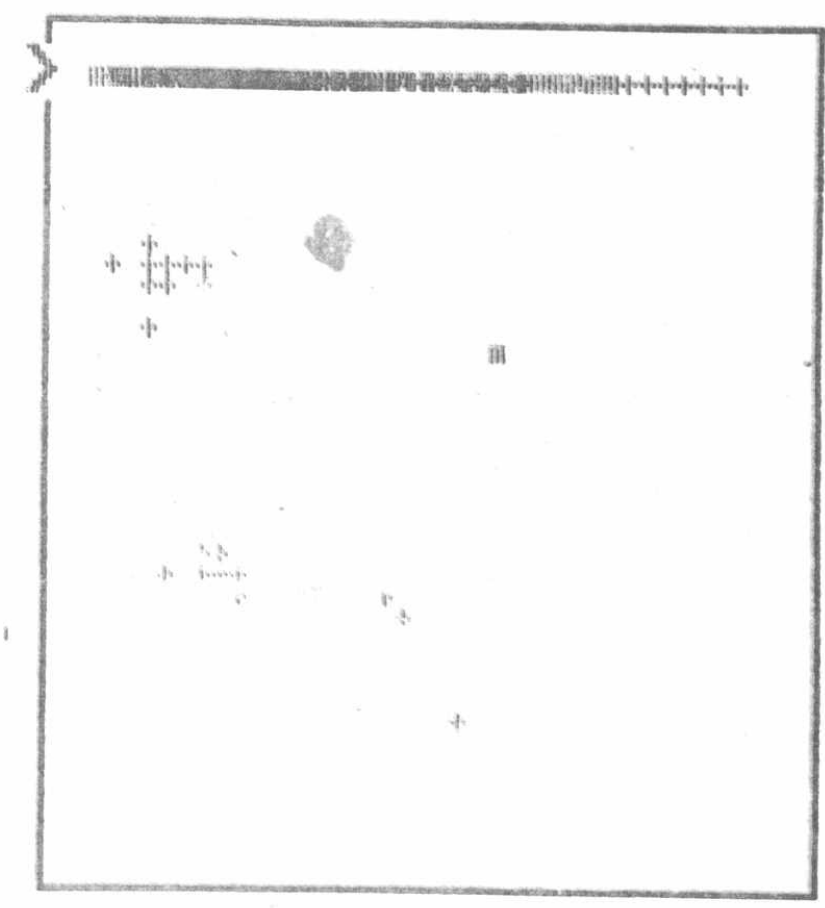

